\title{
THE DIFFRACTION OF A PLANE WAVE THROUGH A GRATING*
}

\author{
BY \\ JOHN W. MILES \\ University of California at Los Angeles
}

Summary. The problem of diffraction and scattering of a normally incident plane wave of sound by an infinite plane grating consisting of infinitely thin, coplanar, equally spaced ( $b$ apart) strips with parallel edges is solved. The potentials on the two sides of the screen are written as Fourier expansions in terms of the velocity in the aperture, and an integral equation for this velocity is determined. An impedance parameter $Z$ whose real part is the transmission coefficient, is defined, and it is shown that the real and imaginary parts of the reciprocal of this parameter may both be specified by variational expressions, which are absolute minima for the solution to the aforementioned integral equation. An alternative formulation, in terms of the pressure discontinuity across the screen, is given, leading to an integral equation and to variational expressions for the real and imaginary parts of $(1-Z)^{-1}$. A solution to the integral equation is given which reduces the problem to the solution of an infinite number of simultaneous equations. It is shown that solving only one of these equations gives a solution which is essentially a solution to Laplace's equation, while if $N$ equations are solved the terms neglected are of the order $N^{-1}\left[\left(1-4 b^{2} / N^{2} \lambda^{2}\right)^{-1 / 2}-1\right]$ or less, where $\lambda$ is the wave length. The solution is extended to the case of a vertically polarized electromagnetic incident wave by direct analogy and to the case of a horizontally polarized electromagnetic wave by a transformation which is a special case of Babinet's principle. The integral equations for an aperture of finite thickness are set up, and an approximate solution including only first order terms in thickness/wavelength is given. Results are given in the form of curves for the transmission coefficient vs. aperture opening for several ratios of grating spacing to wave length less than unity. For the special case of a half open grating the transmission coefficient is plotted out to that value of spacing/wavelength at which the results agree with Kirchhoff's theory.

Introduction. The problem to be solved is the scattering and diffraction of a plane wave of sound which is (essentially) normally incident upon a plane grating consisting of an infinite number of infinitely thin parallel ribbons of width $b-d$ whose centers are spaced a distance $b$ apart, as shown in Fig. 1. Since, by symmetry, the normal velocity must vanish at the planes which form the perpendicular bisectors of the ribbons, shown by dashed lines in Fig. 1, the problem is the same as the calculation of the transmission of sound through a symmetrical window of opening $b-d$ placed perpendicular to two, infinite, parallel plates, a distance $b$ apart. This problem, subject to certain restrictions on the frequency, has been treated by the present author. ${ }^{1}$ For the sake of completeness and in order to investigate further the limitations on the frequency, the analysis will be carried out independently of earlier papers.

The solution will be formulated in terms of the normal velocity in the plane of the grating, which, by virtue of Huygen's principle, may be regarded as a source distribution.

*Received March 18, 1948.

1John W. Miles, The analysis of plane discontinuities in cylindrical tubes, J. Acoust. Soc. Am. 17, Part I: 259-272, Part II: 272-285 (1946). The variational methods used therein were due to J. S. Schwinger. 
The potentials on the two sides of the screen may then be regarded as composed of the solution corresponding to no aperture in the screen (i.e., complete reflection) plus radiation from the unknown source distribution over the aperture. The normal derivatives of the potentials on the two sides of the screen will then automatically satisfy appropriate boundary conditions, while the requirement that the potentials themselves be continuous
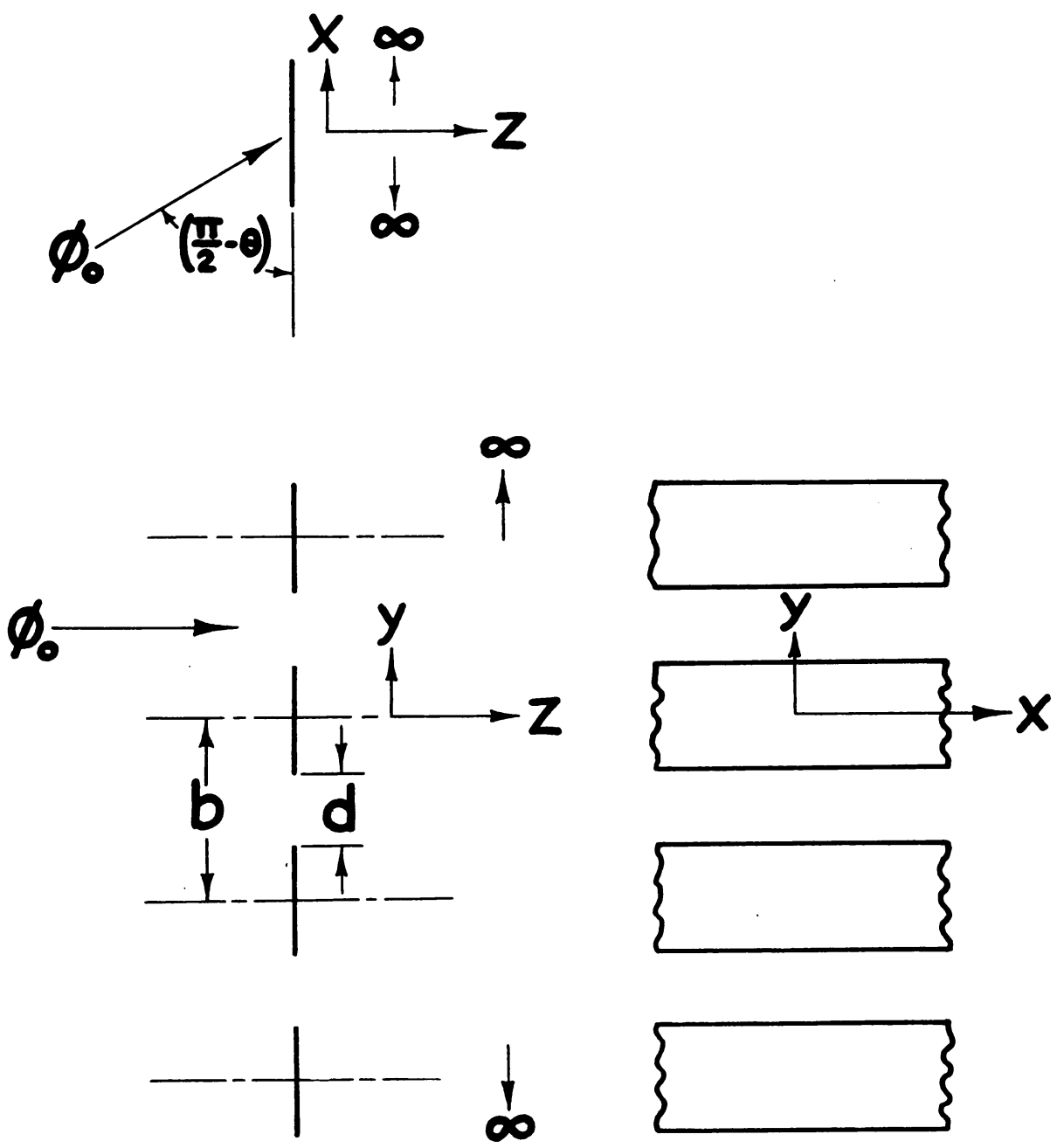

FIg. 1. Three views of infinite grating.

across the aperture yields an integral equation for the determination of the source distribution (or aperture velocity). An alternative formulation in terms of the pressure discontinuity across the screen will also be given.

In many cases, it is sufficient to know the transmission coefficient for the grating, i.e., the ratio of the energy density transmitted through the grating to that in the incident wave. It is convenient to introduce a complex impedance parameter, whose real part is the transmission coefficient, and this leads to a variational formulation of the problem. 
Velocity potentials. Is is known ${ }^{2}$ that if the velocity vector $\mathbf{q}$ is specified as the gradient of a potential $\phi$ and a harmonic time variation $\exp (j \omega t)$ is assumed, the potential satisfies the scalar Helmholtz equation

$$
\begin{gathered}
\nabla^{2} \phi(x, y, z)+k^{2} \phi(x, y, z)=0, \quad \text { (1) } \quad k=\omega / c, \\
\mathbf{q}(x, y, z, t)=\exp (j \omega t) \nabla \phi(x, y, z)
\end{gathered}
$$

while the pressure is given by

$$
p(x, y, z, t)=-j \omega \rho_{0} \exp (j \omega t) \phi(x, y, z) .
$$

If the incident wave from $z=-\infty$ has its wave normal in the $x z$ plane, it may be designated by

$$
\phi_{i n c}(x, y, z)=\Phi_{0} \exp [-j k(x \sin \theta+z \cos \theta)],
$$

where the axes are as shown in Fig. 1. It is evident (since there is no discontinuity in the $x$ direction) that the dependence on the $x$ coordinate, together with the amplitude $\Phi_{0}$, may be removed by writing

$$
\begin{gathered}
\phi(x, y, z)=\Phi_{0} \exp (-j k x \sin \theta) \varphi(y, z), \\
\nabla^{2} \varphi(y, z)+\kappa^{2} \varphi(y, z)=0,
\end{gathered}
$$

where

$$
\kappa=k \cos \theta .
$$

It follows that the solution for $\theta \neq 0$ may be obtained from the solution for $\theta=0$ simply by replacing $k$ in the latter by $k \cos \theta$ and multiplying the result by $\exp (-j k x \sin \theta)$. Accordingly, only the case $\theta=0$ will be discussed in the following sections.

A solution to the foregoing equations which is periodic in $y$, corresponding to the pei iodicity of the grating, which corresponds to the incident wave (5), and which satisfies the boundary conditions

$$
\begin{aligned}
\frac{\partial \varphi}{\partial y}(0, z) & =\frac{\partial \varphi}{\partial y}(b, z)=0, \\
\frac{\partial \varphi}{\partial z}(y, 0) & =0, \quad y \text { in } \tau, \\
& =-j k f(y), \quad y \text { in } \sigma,
\end{aligned}
$$

is given by

$$
\begin{aligned}
& z<0: \varphi_{1}(y, z)=2 \cos k z-k \sum_{n=0}^{\infty} b_{n} \kappa_{n}^{-1} \exp \left(j \kappa_{n} z\right) \cos (n \pi y / b), \\
& z>0: \varphi_{2}(y, z)=\quad k \sum_{n=0}^{\infty} b_{n} \kappa_{n}^{-1} \exp \left(-j \kappa_{n} z\right) \cos (n \pi y / b),
\end{aligned}
$$

${ }^{2}$ P. M. Morse, Vibration and sound, McGraw-Hill, New York, 1936. 


$$
\begin{array}{rlrl}
b_{n} & =\left(\frac{2-\delta_{n}^{0}}{b}\right) \int_{\sigma} f(\eta) \cos (n \pi \eta / b) d \eta, \\
\kappa_{n} & =\left(k^{2}-n^{2} \pi^{2} / b^{2}\right)^{1 / 2} & \text { if } & k>(n \pi / b), \\
& =-j\left(n^{2} \pi^{2} / b^{2}-k^{2}\right)^{1 / 2} & \text { if } & k<(n \pi / b) .
\end{array}
$$

The aperture region is denoted by $\sigma$, while the obstacle is denoted by $\tau$. The leading term $2 \cos k z$ in $\varphi_{1}$ represents the solution corresponding to total reflection and would be the complete solution (i.e., $b_{n} \equiv 0$ ) if the grating were replaced by an unperforated screen. The remaining terms represent the waves which are radiated due to the presence of the aperture $\sigma$, their Fourier amplitudes being chosen to correspond to a normal velocity $-j k f(y)$, as specified by Eq. (9c), in the aperture $\sigma$. The vanishing of this latter velocity on the obstacle $\tau$, as required by Eq. (9b), is ensured by integrating, cf. Eq. (11), only over $\sigma$. The boundary conditions of Eq. (9a) specify the periodicity of the solution and are satisfied by the choice of the expansion functions $\cos (n \pi y / b)$. The behavior of the propagation constants $\kappa_{n}$ at their branch points, cf. Eq. (12), is chosen to ensure attenuation of the non-propagated waves.

The only remaining boundary condition to be satisfied is that of continuity of pressure across the aperture, i.e.,

$$
\varphi_{1}(y, 0)=\varphi_{2}(y, 0), \quad y \text { in } \sigma .
$$

Substituting Eqs. (10) and (11) in Eq. (13) yields the integral equation

$$
\begin{gathered}
\int_{\sigma} K(y, \eta) f(\eta) d \eta=1, \quad y \text { in } \sigma, \\
K(y, \eta)=K(\eta, y)=\sum_{n=0}^{\infty}\left(\frac{2-\delta_{n}^{0}}{b}\right) Y_{n} \cos (n \pi y / b) \cos (n \pi \eta / b), \\
Y_{n}=\left(k / \kappa_{n}\right)=\left[1-(n \lambda / 2 b)^{2}\right]^{-1 / 2}, \quad(2 b / \lambda)>n, \\
=j\left[(n \lambda / 2 b)^{2}-1\right]^{-1 / 2}, \quad(2 b / \lambda)<n .
\end{gathered}
$$

Before discussing the solution of Eq. (14) it will be convenient to discuss alternative formulations of the problem and to introduce an impedance parameter.

Aperture impedance. The energy flow per unit $x$ width through the grating is given by

$$
P=\frac{1}{2} R l \int_{\sigma} \bar{p}(x, y, 0, t) q_{z}(x, y, 0, t) d y .
$$

Substituting $p$ from Eq. (4), $q_{2}$ from Eq. (9c) and observing from Eqs. (10) and (13) that $\varphi=1$ in the aperture, Eq. (17) reduces to

$$
P=\frac{1}{2} \rho_{0} c k^{2} \Phi_{0}^{2} \cos \theta\left[R l \int_{\sigma} f(y) d y\right] .
$$

The aperture impedance will therefore be defined by

$$
Z=R+j X=\frac{1}{b} \int_{\sigma} f(y) d y=b_{0}
$$

where $R$ and $X$ are the real (resistive) and imaginary (reactive) parts of $Z$. 
Now in Kirchhoff's theory ${ }^{3}$ of diffraction it is assumed that the fields in the aperture are the same as they would be in the absence of $\tau$, i.e., $\phi_{1}(x, y, 0)=\phi_{2}(x, y, 0)=$ $\phi_{\text {inc }}(x, y, 0)$, in which case $f(y)=1$. The ratio of actual tramsmilted energy to that predicted by Kirchhoff's theory is therefore given by

$$
P / P_{K}=\left(\frac{d}{b}\right)^{-1}\left[\frac{R l}{b} \int_{\sigma} f(y) d y\right]=\left(\frac{b}{d}\right) R .
$$

The reactance term $X$ is a measure of the non-propagated energy stored in the vicinity of the aperture, i.e. of the standing waves.

It may be remarked that the impedance defined by Eq. (19) is closely analogous in the quantity utilized by Rayleigh in his studies of apertures in cavities. ${ }^{4}$ On the other hand, the impedance differs from that defined in the paper quoted in Footnote 1 in including the "radiation resistance" $R$, and, as will be shown subsequently, also in the definition of the imaginary component.

Variational formulation. Multiplying both sides of Eq. (14) by $f(y) d y$, integrating over the aperture, and dividing through by Eq. (19) yields

$$
Z^{-1}=Y=G+j B=b\left[\int_{\sigma} \bar{f}(y) d y \int_{\sigma} f(\eta) d \eta\right]^{-1} \int_{\sigma} \bar{f}(y) d y \int_{\sigma} f(\eta) K(y, \eta) d \eta .
$$

Equation (21) is a variational equation of a type first studied by Schwinger ${ }^{5}$, although in the present case both the kernel $K$ and the unknown function $f$ are complex. In order to further study the properties of Eq. (21), it is expedient to reduce it to two real expressions. Thus, write

$$
\begin{gathered}
f(y)=f_{1}(y)+j f_{2}(y), \\
K(y, \eta)=K_{1}(y, \eta)+j K_{2}(y, \eta)
\end{gathered}
$$

where $f_{1}$ and $f_{2}$ are both real functions, and $K_{1}$ and $K_{2}$ are the real and imaginary parts of the kernel, corresponding to the $Y_{n}$ of Eqs. $(16 \mathrm{a}, \mathrm{b})$.

Substituting Eqs. (22) and (23) in Eq. (21), remembering the symmetry of the kernel in $(y, \eta)$, yields

$$
\begin{aligned}
& G=b[\left.\left(\int_{\sigma} f_{1} d y\right)^{2}+\left(\int_{\sigma} f d y\right)^{2}\right]^{-1} \\
& \cdot\left[\int_{\sigma} f_{1} d y \int_{\sigma} f_{1} K_{1} d \eta+\int_{\sigma} f_{2} d y \int_{\sigma} f_{2} K_{1} d \eta\right] \\
& B=b\left[\left(\int_{\sigma} f_{1} d y\right)^{2}+\left(\int_{\sigma} f_{2} d y\right)^{2}\right]^{-1} \\
& \cdot\left[\int_{\sigma} f_{1} d y \int_{\sigma} f_{1} K_{2} d \eta+\int_{\sigma} f_{2} d y \int_{\sigma} f_{2} K_{2} d \eta\right] .
\end{aligned}
$$

3J. A. Stratton, Electromagnetic theory, McGraw-Hill, New York, 1941. Since the Kirchhoff calculation is generally carried out by integrating the flux through a hemisphere, the results designated as Kirchhoff herein might more precisely be labelled geometric.

'Lord Rayleigh, Theory of sound, Dover Press, New York, 1946.

${ }^{5}$ At the M. I. T. Radiation Laboratory (1941-1945); see also the paper quoted in Footnote 1. 
Similarly the integral equation (14) may be rewritten:

$$
\begin{aligned}
& \int_{\sigma}\left[K_{1}(y, \eta) f_{1}(\eta)-K_{2}(y, \eta) f_{2}(\eta)\right] d \eta=1 \\
& \int_{\sigma}\left[K_{1}(y, \eta) f_{2}(\eta)+K_{2}(y, \eta) f_{1}(\eta)\right] d \eta=0 .
\end{aligned}
$$

Now by varying $G$ and $B$ as given by Eqs. (24), with respect to both $f_{1}$ and $f_{2}$, it is found that the functions $f_{1}$ and $f_{2}$ which satisfy Eqs. (25) make both $G$ and $B$ stationary. Moreover, from the symmetry of $K_{1}$ and $K_{2}$, both $G$ and $B$ are positive definite forms (since they will be sums of squares), and it follows that they are absolute minima with respect to variations about the true functions $f_{1}$ and $f_{2}$. The formal proof is analogous to that given in Appendix $\mathrm{C}$ of the paper quoted in Footnote 1 for the variational expression obtained by assuming that $f_{1}$ and $f_{2}$ differ only by a constant in Eq. (24b).

A closer analogy to the cases treated in the author's earlier paper ${ }^{1}$ is obtained by assuming $\lambda>b$, as assumed therein, in which case it follows from Eq. (15) that $K_{1}=1$, and Eq. (24a) reduces to

$$
G=1 \quad \text { if } \quad \lambda>b \cos \theta
$$

In this case, it may be assumed that $f_{2}=0$, and it is found that the susceptance $B$ is half that for the symmetrical window mentioned in the Introduction to the present paper.

Alternative formulation. The problem can also be formulated in terms of the pressure discontinuity across the screen, defined by

$$
\gamma(y)=\frac{1}{2}\left[\varphi_{1}\left(y, 0^{-}\right)-\varphi_{2}\left(y, 0^{+}\right)\right] .
$$

In terms of $\gamma(y)$, the potentials may be written

$$
\begin{gathered}
z \lessgtr 0: \varphi_{2}^{1}(y, z)=\exp (-j k z) \pm \sum_{n=0}^{\infty} d_{n} \exp \left( \pm j \kappa_{n} z\right) \cos (n \pi y / b) \\
d_{n}=\left(\frac{2-\delta_{n}^{0}}{b}\right) \int_{\tau} \gamma(\eta) \cos (n \pi \eta / b) d \eta
\end{gathered}
$$

The potentials given by Eq. (28) evidently satisfy Eq. (13) implicitly, since the Fourier coefficients are so defined. Moreover, differentiation shows that the normal velocity is continuous at $z=0$, so that Eq. (9c) is satisfied. It remains to satisfy Eq. (9b) by demanding the integral equation.

$$
\begin{gathered}
\int_{\tau} H(y, \eta) \gamma(\eta) d \eta=1, \quad y \text { in } \tau \\
H(y, \eta)=\sum_{n=0}^{\infty}\left(\frac{2-\delta_{n}^{0}}{b}\right) Z_{n} \cos (n \pi y / b) \cos (n \pi \eta / b), \\
Z_{n}=Y_{n}^{-1}=k^{-1}\left[k^{2}-(n \pi / b)^{2}\right]^{1 / 2} .
\end{gathered}
$$


From Eqs. (9b) and (27) it is evident that $\gamma(y)$ and $f(y)$ are related by.

$$
\begin{aligned}
& f(y)=1-\int_{\tau} H(y, \eta) \gamma(\eta) d \eta, \\
& \gamma(y)=1-\int_{\sigma} K(y, \eta) f(\eta) d \eta,
\end{aligned}
$$

so that the Fourier coefficients of $f$ and $\gamma$ are related by

$$
b_{n}+d_{n}=\delta_{n}^{0} .
$$

For $n=0$, Eqs. (34) and (19) yield

$$
Z=1-\frac{1}{b} \int_{\tau} \gamma(\eta) d \eta
$$

Multiplying both sides of Eq. (30) by $\bar{\gamma}(y) d y$, integrating over the obstacle, and dividing through by $1-Z$ from Eq. (35) yields the variational expression

$$
(1-Z)^{-1}=b\left[\int_{\tau} \bar{\gamma}(y) d y \int_{\tau} \gamma(\eta) d \eta\right]^{-1}\left[\int_{\tau} \bar{\gamma}(y) d y \int_{\tau} \gamma(\eta) H(y, \eta) d \eta\right] .
$$

Now Eq. (36) is exactly analogous to Eq. (21), and it may be shown that its real and imaginary parts are absolute minima with respect to variations of $\gamma(y)$ about the true value of $\gamma(y)$ satisfying the integral equation (30). In order to compare the results so obtained, it may be observed that

$$
(1-Z)^{-1}=\left[(1-G)^{2}+B^{2}\right]^{-1}[(1-G)+j B] .
$$

For the special case where Eq. (26) holds, it is seen that Eq. (36) makes $1 / B$ a minimum, and the results of Eqs. (24b) and (36) therefore bound $B$ from above and below. This case was treated before. ${ }^{1}$ For the case where the wavelength is less than $b$ Eq. (24) bounds $G$ and $B$ from above, while Eq. (36) bounds $\left[(1-G)+(1-G)^{-1} B^{2}\right]$ and $\left[B+B^{-1}(1-\right.$ $G)^{2}$ ] from below.

Solution to the integral equation. In the problem at hand, the most appropriate attack appears to be an approximate solution to the integral equation (14). The accuracy of the solution can then be checked by substituting the solution so obtained in the variational expression (21) and by calculating $\gamma$ from Eqs. (33) and (34) and substituting in Eq. (36).

In order to solve Eq. (14), the first step is to rewrite the kernel $K(y, \eta)$ as

$$
\begin{aligned}
K(y, \eta)=b^{-1} & +j(2 k / \pi) \sum_{n=1}^{\infty} n^{-1} \cos (n \pi y / b) \cos (n \pi \eta / b) \\
& +(2 k / \pi) \sum_{n=1}^{\infty} \Delta_{n} \cos (n \pi y / b) \cos (n \pi \eta / b), \\
\Delta_{n}=n^{-1}\left\{\left[(2 b / n \lambda)^{2}-1\right]^{-1 / 2}-j\right\}, & (2 b / \lambda)>n, \\
=j n^{-1}\left\{\left[1-(2 b / n \lambda)^{2}\right]^{-1 / 2}-1\right\}, & (2 b / \lambda)<n .
\end{aligned}
$$


The second term in Eq. (32) may be written in closed form, since

$$
2 \sum_{n=1}^{\infty} n^{-1} \cos (n z) \cos (n \zeta)=-\ln [2|\cos z-\cos \zeta|],
$$

whence

$$
\begin{array}{r}
K(y, \eta)=b^{-1}-j(k / \pi) \ln [2|\cos (\pi y / b)-\cos (\pi \eta / b)|] \\
+(2 k / \pi) \sum_{n=1}^{\infty} \Delta_{n} \cos (n \pi y / b) \cos (n \pi \eta / b) .
\end{array}
$$

Before substituting Eq. (41) in Eq. (14), it is expedient to introduce the change of variable

$$
\begin{gathered}
\cos (\pi y / b)=\alpha \cos \theta, \quad(42 a) \quad \alpha=\sin (\pi d / 2 b), \\
u(\theta)=\alpha\left(1-\alpha^{2} \cos ^{2} \theta\right)^{-1 / 2} f\left[(b / \pi) \cos ^{-1}(\alpha \cos \theta)\right] \sin \theta .
\end{gathered}
$$

Substituting Eqs. (42) and (43) in Eq. (14) yields

$$
\begin{gathered}
\frac{1}{\pi} \int_{0}^{\pi} b K(\theta, \psi) u(\psi) d \psi=1, \quad 0<\theta<\pi, \\
b K(\theta, \psi)=1-j(k b / \pi) \ln [2 \alpha|\cos \theta-\cos \psi|] \\
\quad+(2 k b / \pi) \sum_{n=1}^{\infty} \Delta_{n} \cos (n \pi y / b) \cos (n \pi \eta / b) \\
=[1-j(k b / \pi) \ln \alpha]+j(2 k b / \pi) \sum_{n=1}^{\infty} n^{-1} \cos n \theta \cos n \psi \\
\quad+(2 k b / \pi) \sum_{n=1}^{\infty} \Delta_{n} \cos (n \pi y / b) \cos (n \pi \eta / b),
\end{gathered}
$$

where $y, \eta$ and $\theta, \psi$ in the last term are understood to be related by Eq. (42). Now expand $u(\theta)$ in the Fourier series

$$
u(\theta)=\sum_{n=0}^{\infty} a_{n} \cos (n \theta) .
$$

Substituting Eq. (46) in Eq. (44) yields

$$
\begin{gathered}
{[1-j(k b / \pi) \ln \alpha] a_{0}+j(k b / \pi) \sum_{n=1}^{\infty} a_{n} n^{-1} \cos (n \theta)} \\
+(k b / \pi) \sum_{r=1}^{\infty} \Delta_{r} \cos (r \pi y / b) \sum_{s=0}^{\infty} a_{s} I_{r s}=1, \\
I_{r s}=\frac{2}{\pi} \int_{0}^{\pi} \cos \left[r \cos ^{-1}(\alpha \cos \psi)\right] \cos (s \psi) d \psi .
\end{gathered}
$$


Moreover, since $I_{m n}$ are the Fourier expansion coefficients of $\cos (n \pi y / b)$, Eq. (47) may be rewritten

$$
\begin{aligned}
& {[1-j(k b / \pi) \ln \alpha] a_{0}+j(k b / \pi) \sum_{n=1}^{\infty} a_{n} n^{-1} \cos (n \theta)} \\
& \quad+(k b / \pi) \sum_{r=1}^{\infty} \Delta_{r} \sum_{n=0}^{\infty}\left(1+\delta_{n}^{0}\right)^{-1} I_{r n} \cos (n \theta) \sum_{s=0}^{\infty} a_{s} I_{r s}=1 .
\end{aligned}
$$

Now, since Eq. (49) must be valid for all $\theta$ between 0 and $\pi$, it follows that

$$
\begin{aligned}
& \delta_{0}^{n}[1-j(k b / \pi) \ln \alpha] a_{0}+(k b / \pi)\left\{j\left(1-\delta_{n}^{0}\right) n^{-1} a_{n}\right. \\
& \left.\quad+\sum_{r=1}^{\infty}\left(1+\delta_{n}^{0}\right)^{-1} \Delta_{r} I_{r n} \sum_{s=0}^{\infty} I_{r s} a_{s}\right\}=\delta_{n}^{0}, \quad n=0,1,2, \cdots
\end{aligned}
$$

which is an infinite set of simultaneous equations for the determination of the $d_{n}$.

While it would have been simpler to solve the integral equation (12) by a direct Fourier expansion in $\cos (n \pi y / b)$, leading to a set of simultaneous equations for the Fourier coefficients, the transformation to the $\theta$ coordinate allows the separation of the $\ln \alpha$ term, which is essentially the susceptance in the limit of zero frequency.

The Irs integrals may be easily evaluated by an identity due to $\mathrm{Jacobi}^{6}$

$$
\int_{0}^{\pi} \phi(\cos \theta) \cos (n \theta) d \theta=[1 \cdot 3 \cdots(2 n-1)]^{-1} \int_{0}^{\pi} \frac{d^{n} \phi(\cos \theta)}{d(\cos \theta)^{n}}(\sin \theta)^{2 n} d \theta .
$$

Now

$$
\begin{gathered}
\cos (r \pi \eta / b)=\sum_{p=0}^{r} c_{p}^{r} \alpha^{p}(\cos \theta)^{p}, \\
c_{p}^{r}=\text { coeff. of }(\cos x)^{p} \text { in expansion of } \cos (n x),
\end{gathered}
$$

whence it follows that

$$
\begin{aligned}
\frac{d^{s} \cos (r \pi \eta / b)}{d(\cos \theta)^{s}} & =\sum_{p=s}^{r} p(p-1) \cdots(p-s+1) c_{p}^{r} \alpha^{p}(\cos \theta)^{p-n}, & & s \leq r \\
& =0 \quad, & & r<s
\end{aligned}
$$

Using the result

$$
\int_{0}^{\pi}(\cos \theta)^{m}(\sin \theta)^{n} d \theta=B\left(\frac{m+1}{2}, \frac{n+1}{2}\right)
$$

where $B$ is the Beta function, it follows that

$$
\begin{aligned}
I_{r s}= & \frac{2}{\pi}[1 \cdot 3 \cdots(2 s-1)]^{-1} \sum_{p=s}^{r} \\
& \quad p(p-1) \cdots(p-s+1) c_{p}^{r} \alpha^{p} B\left(\frac{p-s+1}{2}, \frac{2 s+1}{2}\right), \quad r \geq s, \\
= & 0, \quad r<s .
\end{aligned}
$$

${ }^{6} \mathrm{H}$. Bateman, Partial differential equations, Dover Press, New York, 1944, p. 463. (Note that the first integral given by Bateman on the top of p. 464 is incorrect and is not used here.) 
In the present case, due to symmetry, only even values of $r$ and $s$ need be considered, so that $p-s$ and $2 s$ will be even, in which case

$$
\frac{2}{\pi} B\left(\frac{p-s+1}{2}, \frac{2 s+1}{2}\right)=\left[2^{p+s-1}\left(\frac{p-s}{2}\right) !\left(\frac{p+s}{2}\right) ! s !\right]^{-1}(p-s) !(2 s) !
$$

In an approximate solution, only a finite number, say $N$, of Eqs. (50) will be solved. The error in solving $N$ equations will evidently be of order $\Delta_{N}$ at worst. While the $\Delta_{n}$ (cf. Eq. 39) generally decrease with increasing $n$, each possesses a singularity at $b / \lambda=n / 2$, so that it will generally be necessary to satisfy the inequality

$$
N>(2 b / \lambda) \text {. }
$$

It should also be remembered that, due to symmetry, the odd $A_{n}$ vanish identically, and the number of equations to be solved for a given $N$ is actually only $(N+1) / 2$.

Electromagnetic problem. The case of a plane electromagnetic wave normally incident on a grating (assumed perfectly conducting at radio frequencies or perfectly reflecting at optical frequencies) is analogous to the above acoustical problem since, due to the normal incidence, it is possible to derive all six components of the electromagnetic field from a single component of the incident field, provided that the cases of vertical (electric field) and horizontal polarization of the incident wave are treated separately.

Vertically polarized wave. Consider first the vertically polarized incident wave consisting of the fields

$$
\begin{aligned}
\mathbf{H}_{0} & =\mathbf{i} H_{x}^{0} \exp (-j k z), \\
\mathbf{E}_{0} & =-\mathbf{j} H_{x}^{0} \exp (-j k z),
\end{aligned}
$$

which satisfy Maxwell's equations (MKS units)

$$
\begin{aligned}
\nabla \times \mathbf{E} & =-j k \mathbf{H}, \\
\nabla \times \mathbf{H} & =j k \mathbf{E}, \\
\nabla \cdot \mathbf{E} & =0, \\
\nabla \cdot \mathbf{H} & =0 .
\end{aligned}
$$

The boundary conditions to be satisfied in the screen are

$$
E_{x}=E_{y}=H_{z}=0
$$

only two of which are independent, according to Eqs. (59). Finally, each of the field components satisfies the scalar Helmholtz equation (1), which follows directly from Maxwell's equations (59) and (60).

Now in the case of the incident wave (58), it is evident that the entire problem may be formulated in terms of field components $H_{x}, E_{y}$, and $E_{z}$. Moreover, from Eqs. (59), it is seen that

$$
\begin{aligned}
& j k E_{y}(y, z)=\frac{\partial H_{x}}{\partial z}(y, z), \\
& j k E_{z}(y, z)=-\frac{\partial H_{x}}{\partial y}(y, z),
\end{aligned}
$$


which satisfy Eqs. (59) and (60). Therefore, it is evident from comparing Eqs. (5) and (58a), (9b) and (61b), and (9c) and (61b) that

$$
\begin{aligned}
H_{x}^{0} & \sim \Phi_{0}, \\
H_{x}(y, z) & \sim \Phi_{0} \varphi(y, z), \\
E_{y}(y, 0) & \sim \Phi_{0} f(y) .
\end{aligned}
$$

It follows that the foregoing acoustical problem yields a direct solution to the electromagnetic problem when the incident wave is plane and vertically polarized. (It may also be shown that the pressure discontinuity $\gamma$ is analogous to the current induced in the screen by the magnetic field.)

Horizontally polarized wave. A horizontally polarized incident field consists of the components

$$
\begin{aligned}
\mathbf{E}_{0} & =\mathbf{i} E_{x}^{0} \exp (-j k z), \\
\mathbf{H}_{0} & =\mathbf{j} E_{x}^{0} \exp (-j k z) .
\end{aligned}
$$

Consider now the solution

$$
\begin{aligned}
& z \lessgtr 0: E_{x}^{1_{2}}(y, z)=E_{x}^{0}\left\{\exp (-j k z)-k \sum_{n=0}^{\infty} b_{n} \kappa_{n}^{-1} \exp \left( \pm j \kappa_{n} z\right) \cos (n \pi y / b)\right\}, \\
& z \lessgtr 0: H_{y}^{1_{2}}(y, z)=E_{x}^{0}\left\{\exp (-j k z) \pm \sum_{n=0}^{\infty} b_{n} \exp \left( \pm j \kappa_{n} z\right) \cos (n \pi y / b)\right\} .
\end{aligned}
$$

It is evident that Eq. (65) corresponds to Eq. (64) and satisfies Eqs. (59) and (60). Moreover, if $f(y)$ is a solution to Eq. (14) in the plane $z=0$, the following conditions are satisfied:

$$
\begin{array}{rlrl}
E_{x}^{1}(y, 0) & =E_{x}^{2}(y, 0) \quad, \quad 0 \leq y \leq b, \\
E_{x}^{1}(y, 0) & =E_{x}^{2}(y, 0)=0, \quad & & y \text { in } \sigma, \\
H_{y}^{1}(y, 0) & =H_{y}^{2}(y, 0)=E_{x}^{0}, & & y \text { in } \tau, \\
H_{y}^{1}\left(y, 0^{-}\right)-H_{y}^{2}\left(y, 0^{+}\right) & =2 E_{x}^{0} f(y) \quad, \quad & & y \text { in } \sigma .
\end{array}
$$

Hence, it follows that Eq. (65) represents a solution to Maxwell's equations which satisfies boundary conditions appropriate to the complementary grating, i.e., that grating obtained by interchanging aperture $\sigma$ and obstacle $\tau$ of the original grating. This result is a special case of Babinet's principle, which, in its rigorous form, has been discussed by Copson $^{7}$, following Booker, by Watson ${ }^{8}$, and by Schwinger ${ }^{9}$.

From Eq. (66d) it is evident that $f(y)$ in the present case represents the current flowing in the screen, whereas in the case of the vertically polarized incident wave $f(y)$ represented the tangential electric field in the aperture. Conversely, it may be observed that an alternative solution for the present case is given by

${ }^{7} \mathrm{E}$. T. Copson, An integral equation method of solving plane diffraction problems. Proc. Roy. Soc. London (A) 186, 100-118 (1946).

${ }^{8} \mathrm{~W}$. H. Watson, Physical principles of waveguide transmission, Clarendon Press, Oxford, 1947.

${ }^{9}$ J. Schwinger, M. I. T. Radiation Lab. Reports, Cambridge, Mass., 1941-1945. 


$$
\begin{aligned}
& z<0: E_{x}^{1}(y, z)=E_{x}^{0}\left\{-2 j \sin (k z)+\sum_{n=0}^{\infty} d_{n} \exp \left(j \kappa_{n} z\right) \cos (n \pi y / b)\right\}, \\
& z>0: E_{x}^{2}(y, z)=E_{x}^{0} \sum_{n=0}^{\infty} d_{n} \exp \left(-j \kappa_{n} z\right) \cos (n \pi y / b), \\
& z<0: H_{y}^{1}(y, z)=E_{x}^{0}\left\{2 \cos (k z)-k^{-1} \sum_{n=0}^{\infty} d_{n} \kappa_{n} \exp \left(j \kappa_{n} z\right) \cos (n \pi y / b)\right\}, \\
& z>0: H_{y}^{2}(y, z)=E_{x}^{0} k^{-1} \sum_{\cdot n=0}^{\infty} d_{n} \kappa_{n} \exp \left(-j \kappa_{n} z\right) \cos (n \pi y / b) .
\end{aligned}
$$

The boundary conditions (66a) and (66b) are automatically satisfied by Eqs. (67a,b), since

$$
E_{x}^{1}(y, 0)=E_{x}^{2}(y, 0)=E_{x}^{0} \gamma(y)
$$

The boundary condition (66c) is satisfied if $\gamma(y)$ is a solution to the integral equation (30). Hence, the aperture field in the horizontally polarized problem is equivalent to the screen current in the complementary problem (vertically polarized incident wave and complementary grating). This result is another aspect of Babinet's principle.

Transmission and reflection coefficients. If the transmission coefficient is defined as the ratio of the energy flow to the right of the screen to the energy flow in the undisturbed incident wave, it follows from Eqs. (18) and (19) that

$$
T=R l(Z)=R l\left(a_{0}\right)
$$

for the acoustic problem.

In the electromagnetic problem the transmission coefficient is given by integrating Poynting's vector over the aperture and dividing by the energy flow (between $y=0, b$ ) in the incident wave to obtain

$$
\begin{aligned}
T_{\text {。 }} & =b^{-1}\left(H_{x}^{0}\right)^{-2} R l \int_{\sigma} \mathbf{E}(y, 0) \times \overline{\mathbf{H}}(y, 0) d y, \\
& =b^{-1} R l \int_{\sigma} f(y) d y=R l(Z)=R l\left(a_{0}\right)
\end{aligned}
$$

for the vertically polarized case. For the case of horizontal polarization

$$
\begin{aligned}
T_{h} & =b^{-1}\left(E_{x}^{0}\right)^{-2} R l \int_{\tau} \mathbf{E}(y, 0) \times \overline{\mathbf{H}}(y, 0) d y \\
& =b^{-1} R l \int_{\tau} \gamma(y) d y \\
& =R l\left[1-b^{-1} \int_{\sigma} f(y) d y\right] \\
& =1-T_{v} .
\end{aligned}
$$


The relation $T_{h}+T_{0}=1$ wbere $T_{h}$ and $T_{0}$ are the transmission coefficients for complementary gratings and complementary polarization, is predicted by Babinet's principle. ${ }^{8}$

From conservation of energy, it is evident that the reflection coefficients are given by $1-T$. In the case where $\lambda>b$, only a plane wave will be reflected, and there will be a simple standing wave pattern to the left of the screen. In this case, as may be seen from Eq. (10), it is convenient to define the complex reflection coefficient $1-b_{0}$, which will then give the phase of the standing waves.

Thick grating. Experimental measurements (electromagnetic) in wave guides ${ }^{10}$ have shown that the principal errors in theoretically calculated diffractions through thin windows, etc. are due to the assumption of zero thickness and that a thickness of as little as 0.005 wavelength may cause a deviation of several per cent between theory and experiment, although the experimental results approach those predicted by theory as thickness is decreased. Lord Rayleigh ${ }^{4}$ predicted that thickness would also be quite important in the acoustical problem, although he does not offer any direct reason or cite any supporting experimental evidence. Moreover, the deviations from an ideal fluid in acoustical experiments are such as to make precision measurements more difficult than in analogous electromagnetic problems.

Consider the window of finite thickness $t$ and symmetrically located opening $d$ between two parallel plates a distance $b$ apart, as shown in Fig. 2. The faces of the window will be

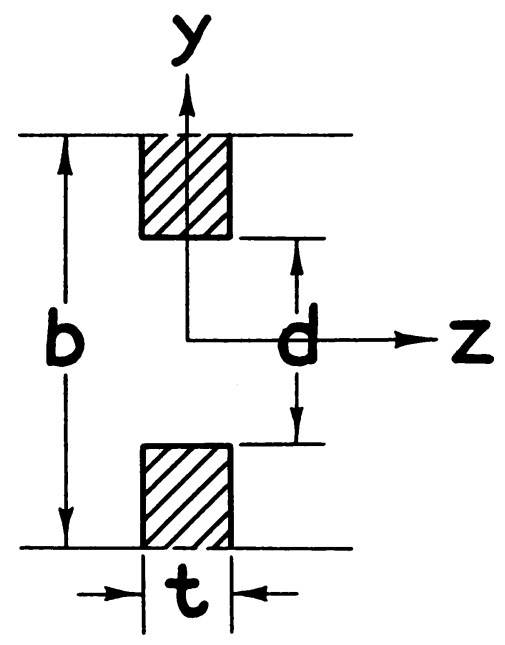

FIG. 2. Section of thick grating.

assumed to lie in the planes $z= \pm t / 2$. The potentials in the three $z$ regions, corresponding to the incident wave (5) are given by

$$
\begin{aligned}
z<-t / 2: \varphi_{1}(y, z)=2 \exp (j k t / 2) \cos [k(z+t / 2)] & \\
& \quad-k \sum_{n=0}^{\infty} b_{n}^{1} \kappa_{n}^{-1} \exp \left[j \kappa_{n}(z+t / 2)\right] \cos (n \pi y / b),
\end{aligned}
$$

${ }^{10}$ Waveguide handbook. M. I. T. Radiation Laboratory Report, $41-1 / 23 / 45$ to be published by McGraw-Hill, New York. 


$$
\begin{aligned}
\left.-t / 2<z<+t / 2: \varphi_{12} y, z\right)=-j k & \sum_{n=0}^{\infty}\left(\frac{2-\delta_{n}^{0}}{d}\right) \zeta_{n}^{-1}\left\{\left(\sin \left[\zeta_{n}(z+t / 2)\right]\right.\right. \\
& \left.+\cot \left(\zeta_{n} t\right) \cos \left[\zeta_{n}(z+t / 2)\right]\right) \int_{\sigma} f_{1}(\eta) \\
& \times \cos \left[(n \pi / d)\left(\eta-\frac{b-d}{2}\right)\right] d \eta \\
& +\left(\sin \left[\zeta_{n}(z-t / 2)\right]-\cot \left(\zeta_{n} t\right) \cos \left[\zeta_{n}(z-t / 2)\right]\right) \\
& \left.\times \int_{\sigma} f_{2}(\eta) \cos \left[(n \pi / d)\left(\eta-\frac{b-d}{2}\right)\right] d \eta\right\} \\
& \times \cos \left[(n \pi / d)\left(y-\frac{b-d}{2}\right)\right], \\
z>t / 2: \varphi_{2}(y, z)=k \sum_{n=0}^{\infty} b_{n}^{2} \kappa_{n}^{-1} \exp \left[-j \kappa_{n}(z-t / 2)\right] \cos (n \pi y / b) & \\
\zeta_{n}=\left[k^{2}\right. & \left.-(n \pi / d)^{2}\right]^{1 / 2} .
\end{aligned}
$$

The $b_{n}^{1,2}$ are given by substituting $f_{1,2}$ in Eq. (11).

The expansion functions and coefficients in Eq. (72) have been chosen so as to satisfy the boundary condition that the normal velocity vanish at all walls, and reduce to the normal velocities in the two apertures, $-j k f_{1}(y)$ and $-j k f_{2}(y)$ at $z=\mp t / 2$ respectively. These velocities are then determined by the requirement that $\varphi_{1}, \varphi_{12}$, and $\varphi_{2}$ be continuous across the two apertures, which yields the simultaneous integral equations

$$
\begin{gathered}
\int_{\sigma}\left[K_{11}(y, \eta) f_{1}(\eta)+K_{12}(y, \eta) f_{2}(\eta)\right] d \eta=\exp (-j k t / 2) \quad y \text { in } \sigma \\
\int_{\sigma}\left[K_{21}(y, \eta) f_{1}(\eta)+K_{22}(y, \eta) f_{2}(\eta)\right] d \eta=0 \\
K_{11}(y, \eta)=K_{22}(y, \eta)=\frac{1}{2} \sum_{n=0}^{\infty}\left\{\left(\frac{2-\delta_{n}^{0}}{b}\right)\left(k / \kappa_{n}\right) \cos (n \pi y / b) \cos (n \pi \eta / b)\right. \\
-j\left(\frac{2-\delta_{n}^{0}}{d}\right)\left(k / \zeta_{n}\right) \cot \left(\zeta_{n} t\right) \cos \left[(n \pi / d)\left(y-\frac{b-d}{2}\right)\right] \\
\left.\times \cos \left[(n \pi / d)\left(\eta-\frac{b-d}{2}\right)\right]\right\}, \\
K_{12}(y, \eta)=K_{21}(y, \eta)=\left(\frac{j}{2}\right) \sum_{n=0}^{\infty}\left(\frac{2-\delta_{n}^{0}}{d}\right)\left(k / \zeta_{n}\right) \csc \left(\zeta_{n} t\right) \\
\quad \times \cos \left[(n \pi / d)\left(y-\frac{b-d}{2}\right)\right] \cos \left[(n \pi / d)\left(\eta-\frac{b-d}{2}\right)\right] .
\end{gathered}
$$


The integral equations (73) can be solved systematically by expanding $f_{1}$ and $f_{2}$ in the functions $\cos [(n \pi / d)\{\eta-(b-d) / 2\}]$, multiplying the integral equations by $\cos [(m \pi / d)$ $\cdot\{y-(b-d) / 2\}] d y$, and integrating over the aperture to obtain a doubly infinite set of simultaneous coefficients for the determination of the expansion coefficients. However, only a simple approximate solution will be given herein, since the effect contemplated is presumably small.

It will be assumed that the fields $f_{1,2}$ are similar to the solution for $t=0$ but differ in phase by an amount proportional to $t / \lambda$, viz:

$$
f_{1,2}(y)=\left[1+j \epsilon_{1,2} k t+\delta_{1,2}(k t)^{2}\right] f_{0}(y)+0\left[(t / \lambda)^{3}\right]
$$

where $f_{0}$ is presumed to be a solution of the integral equation (14). The terms in $\dot{\delta}_{1,2}$ will be dropped in the final results, but it is necessary to include them in the intermediate steps. It will also be assumed that $\epsilon_{1,2}$ are constants, although this is not strictly correct (even up to terms of order $t / \lambda$ ), since the nature of the singularity at the edge of the aperture is quite sensitive to thickness.

The kernel functions of Eq. (74) may be rewritten

$$
\begin{aligned}
& K_{11}(y, \eta)= \frac{1}{2} K_{0}(y, \eta)-j(2 k t)^{-1} K_{12}^{\prime}(y, \eta)+O\left[(t / \lambda)^{3}\right], \\
& K_{12}(y, \eta)= j(2 \kappa t)^{-1} K_{12}^{\prime}(y, \eta)+O\left[(t / \lambda)^{3}\right], \\
& K_{12}^{\prime}(y, \eta)=\sum_{n=0}^{\infty}\left(\frac{2-\delta_{n}^{0}}{d}\right)\left(k / \zeta_{n}\right)^{2} \cos \left[(n \pi / d)\left(y-\frac{b-d}{2}\right)\right] \\
& \quad \times \cos \left[(n \pi / d)\left(\eta-\frac{b-d}{2}\right)\right]
\end{aligned}
$$

where the terms of order $t / \lambda$ can be shown to vanish identically. $K_{0}$ is the kernel of Eq. (14).

Substituting Eqs. (75) and (76) in Fqs. (73), and recalling that $f_{0}$ satisfies Eq. (14), Eqs. (73) reduce to

$$
\begin{aligned}
\frac{1}{2}\left(1+j k t \epsilon_{1}\right) & +\frac{1}{2}\left(\epsilon_{1}-\epsilon_{2}\right) \int_{\sigma} K_{12}^{\prime} f_{0} d \eta-\frac{1}{2} j k t\left(\delta_{1}-\delta_{2}\right) \int_{\sigma} K_{12}^{\prime} f_{0} d \eta \\
& +O\left[(t / \lambda)^{3}\right]=1+\frac{1}{2} j k t, \\
\frac{1}{2}\left(1+j k t \epsilon_{2}\right) & -\frac{1}{2}\left(\epsilon_{1}-\epsilon_{2}\right) \int_{\sigma} K_{12}^{\prime} f_{0} d \eta+\frac{1}{2} j k t\left(\delta_{1}-\delta_{2}\right) \int_{\sigma} K_{12}^{\prime} f_{0} d \eta \\
+ & O\left[(t / \lambda)^{3}\right]=0 .
\end{aligned}
$$

Equating powers of $t$ in Eq. (77a) yields

$$
\left(\epsilon_{1}-\epsilon_{2}\right) \int_{\sigma} K_{12}^{\prime} f_{0} d \eta=1,
$$


while adding Eqs. (77a) and (77b) yields

$$
\epsilon_{1}+\epsilon_{2}=1 \text {. }
$$

Multiplying Eq. (78) by $d y$ and integrating over the aperture $\sigma$ yields (cf. Eq. 19):

$$
\left(\epsilon_{1}-\epsilon_{2}\right) d^{-1} \int_{\sigma} f_{0} d \eta=\left(\epsilon_{1}-\epsilon_{2}\right)(b / d) Z=1 .
$$

Solving Eqs. (79) and (80) yields

$$
\epsilon_{1,2}=\frac{1}{2}[1 \pm(d / b) Y]
$$

where $Y$ is the admittance computed for zero thickness.

The net change in the transmission coefficient is evidently given by

$$
\begin{aligned}
T(t) / T(0) & =\left|1+j k t \epsilon_{2}\right|^{2} \\
& =|1+j(k t / 2)-j(k t / 2)(d / b)(G+j B)|^{2} \\
& =1+(d / b)(k t) B+O\left[(t / \lambda)^{2}\right]
\end{aligned}
$$

where $B$ is the susceptance calculated at $t=0$.

Actually, the correction given by the foregoing approximate analysis is generally too small. This is probably due to the fact that the nature of the singularity near the edge of the aperture is extremely sensitive to thickness, whereas the fields in Eq: (75) possess the same singularity as in the case of the infinitely thin aperture. In order to take this factor into account, one might solve the static problem $k=0$ by conformal mapping and establish a thickness correction based on the difference between the field so obtained and its limiting form at zero thickness. Unfortunately, the results obtained are rather too complex to be useful.

Uniqueness of solution. Bouwkamp, ${ }^{11}$ in reviewing a recent paper by Copson, ${ }^{7}$ has pointed out that solutions to Maxwell's equations such as Copson's, ${ }^{7}$ and also Bethe's, ${ }^{12}$ are not unique inasmuch as they neglect the possibility of a free charge on the edge of the aperture, wiih its resulting scalar potential. The present analysis is open to the same criticism. Unfortunately, a rigorous discussion is complicated by the singularity at the edge of the aperture. Moreover, the results predicted by Bethe's theory ${ }^{12}$ have been verified $^{13}$ with a precision which appears to settle the question from a practical point of view, at least insofar as the distant fields are concerned. It must be remarked, however, that the charge distribution suggested by Bouwkamp might well affect the distribution of the field in the aperture without markedly affecting the field at a distance, since the latter depends primarily on the mean value of the former and not on its distribution. Andrews ${ }^{14}$ has recently made some precision measurements on the electromagnetic field in a circular aperture which might prove to be a valuable confirmation of the theoretically

${ }^{11}$ C. J. Bouwkamp, Math. Revs. 8, 180 (1947).

${ }^{12} \mathrm{H}$. A. Bethe, Theory of diffraction by small holes, Phys. Rev. 66, 163-182 (1944).

${ }^{13}$ Loc. cit., ${ }^{10}$, and other M. I. T. Radiation Laboratory reports of groups 41 and 43.

${ }^{14}$ C. L. Andrews, Diffraction pattern of a circular aperture at short distances, Phys. Rev. 71, 777-781 (1947). 
calculated field in the aperture. Unfortunately, existing solutions for this problem ${ }^{12}{ }^{15}$ are not sufficiently accurate (for holes whose dimensions are comparable to the wavelength) to warrant comparison. Moreover, the fields in the immediate vicinity of the aperture will almost certainly be quite sensitive to thickness.

Numerical results. The $I_{r s}$ computed from Eqs. (55) and (56) for use up to $r=$ $s=6$ are given by

$\begin{array}{ccccc}r / s & 0 & 2 & 4 & 6 \\ 0 & 2 & 0 & 0 & 0 \\ 2 & {\left[2\left(\alpha^{2}-1\right)\right]} & \alpha^{2} & 0 \\ 4 & {\left[2\left(3 \alpha^{2}-1\right)\left(\alpha^{2}-1\right)\right]} & {\left[4 \alpha^{2}\left(\alpha^{2}-1\right)\right]} & \alpha^{4} & 0 \\ 6 & {\left[2\left(10 \alpha^{4}-8 \alpha^{2}+1\right)\left(\alpha^{2}-1\right)\right]} & {\left[3 \alpha^{2}\left(5 \alpha^{2}-3\right)\left(\alpha^{2}-1\right)\right]} & {\left[6 \alpha^{4}\left(\alpha^{2}-1\right)\right]} & \alpha^{6} .\end{array}$

Choosing $N=6$ in Eq. (5), $a_{0}, a_{2}$, and $a_{4}$ are given by

$$
\begin{gathered}
a_{0}^{-1}=1+2 j(b / \lambda) \ln \left(\alpha^{-1}\right)+4(b / \lambda)\left(1-\alpha^{2}\right)^{2} D^{-1}\left\{\Delta_{2}\right. \\
\left.+\left(3 \alpha^{2}-1\right)^{2} \Delta_{4}-6 j \alpha^{4}\left(\alpha^{4}-2 \alpha^{2}+3\right) \Delta_{2} \Delta_{4}\right\} \\
D=1-2 j \alpha^{4}\left[\Delta_{2}+2\left(9 \alpha^{4}-16 \alpha^{2}+8\right) \Delta_{4}\right]-8 \alpha^{12} \Delta_{2} \Delta_{4}, \\
\left(a_{2} / a_{0}\right)=4 \alpha^{2}\left(1-\alpha^{2}\right) D\left\{j\left[\Delta_{2}-4\left(1-\alpha^{2}\right)\left(3 \alpha^{2}-1\right) \Delta_{4}\right]+4 \alpha^{8} \Delta_{2} \Delta_{4}\right\} \\
\left(a_{4} / a_{0}\right)=8 \alpha^{4}\left(1-\alpha^{2}\right) D \Delta_{4}\left[j\left(3 \alpha^{2}-1\right)+4 \alpha^{6} \Delta_{2}\right] \quad(b / \lambda)<3
\end{gathered}
$$

For $\lambda \gg b$, the simplest approximation is given by setting $\Delta_{2}=\Delta_{4}=0$ in Eq. (84a) to obtain*

$$
R l\left(a_{0}\right)=\left\{1+4(b / \lambda)^{2} \ln ^{2}[\csc (\pi d / 2 b)]\right\}^{-1} \quad(b / \lambda) \ll 1
$$

A more accurate expression is given by retaining $\Delta_{2}$, as given by Eq. (39b) to obtain

$$
\begin{gathered}
R l\left(a_{0}\right)=\left\{1+4(b / \lambda)^{2}(\ln [\csc (\pi d / 2 b)]+\Gamma)^{2}\right\}^{-1}, \quad(b / \lambda)<1, \\
\Gamma=\left\{1+\left(\left[1-(b / \lambda)^{2}\right]^{-1 / 2}-1\right) \sin ^{4}(\pi d / 2 b)\right\}^{-1} \\
\times\left\{\left[1-(b / \lambda)^{2}\right]^{-1 / 2}-1\right\} \cos ^{4}(\pi d / 2 b) .
\end{gathered}
$$

${ }^{15}$ Lord Rayleigh, On the passage of waves through apertures in plane screens and allied problems, Philos. Mag. 43, 259-272 (1897) and Sci. Papers 4, 283-296; also, On the incidence of aerial and electric waves upon small obstacles in the form of ellipsoids or electric cylinders, and on the passage of electric waves through a circular aperture in a conducting screen, Philos. Mag. 44, 28-52 (1897) and Sci. Papers 4, 305-327.

*Since this paper was written the author has found that the result given in equation 85 was first found by Horace Lamb, On the diffraction in transmission of electric waves by a metallic grating, Proc. London Math. Society 29, 523-544 (1898). Moreover a bibliography on problems related to this, such as diffraction through a grating of wires, etc. is given by $\mathrm{H}$. Bateman, Electrical and optical wave motion, Cambridge University Press, 1915, p. 89. 
For $(b / \lambda)>1$, the expression for $R l\left(a_{0}\right)$ became rather too complex to be worth writing out explicitly, and it is simpler to calculate $a_{0}$ directly from Eq. (84a).

The result given in Eq. (86) for $b<\lambda$ of course reduces to Eq. (85) for small values of $b / \lambda$. Now, where $b / \lambda$ was less than unity, the accuracy of the susceptance, as given by Eq. (24b) was investigated ${ }^{1}$ by using the variational formulations of Eqs. (29b) and (36), and the susceptance corresponding to the result given by Eq. (86a) was found to be in error by about $0.1 \%$ for $d / b=1 / 2$ and $b / \lambda=1 / 2$, while the susceptance corresponding to the approximation of Eq. (85) was in error by $10 \%$ for these figures. For $d / b=1 / 2$ and $b / \lambda=1$ the approximation corresponding to Eq. (86a) is in error about $1 \%$, while that corresponding to Eq. (85) is in error by over $74 \%$.

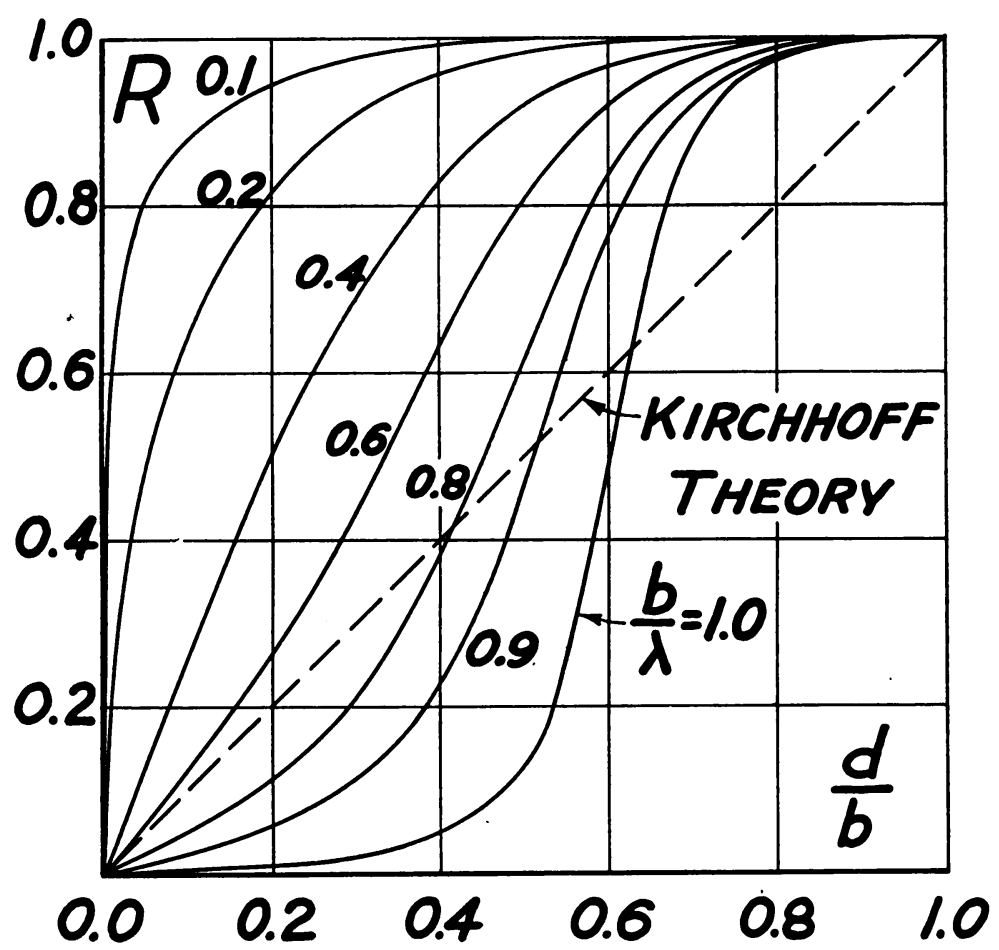

Frg. 3. Resistive component of grating impedance (equivalent to transmission coefficient).

In Figs. 3 and $4 R$ and $X$ are plotted vs. the aperture ratio $d / b$ for $b / \lambda=0,0.1,0.2$, $0.4,0.6,0.8,0.9$, and 1.0 , using, for the most part, data previously calculated ${ }^{10}$. In Fig. 5, $R$ is plotted vs $b / \lambda$ from 0 to 1.9 , for $d / b=1 / 2$, the calculations having been made for $N=4$.

The result obtained by substituting $f(y)=$ const. in Eq. (21) is

$$
G+j B=1+2 \sum_{n=1}^{\infty}\left[1-(n \lambda / b)^{2}\right]^{-1 / 2}(n \pi d / b)^{-2} \sin ^{2}(n \pi d / b)
$$

and is also plotted in Fig. 5 for values of $b / \lambda$ up to 3.8. The accuracy of the transmission factor corresponding to Eq. (87) may be estimated by comparing with the results ob- 
tained from the solution (84) which are accurate to better than $1 \%$ (estimated) for $b / \lambda<2$. The accuracy of Eq. (87) will, however, improve with increasing values of $b / \lambda$.

Moreover, for large values of $b / \lambda$, the imaginary part of Eq. (87) may be neglected, while the real part is given approximately by

$$
\begin{gathered}
G=1+2 \sum_{n=1}^{\infty}(n \pi d / b)^{-2} \sin ^{2}(n \pi d / b)=1+(b / \pi d)^{2} S(\pi d / b), \\
S(x)=\sum_{n=1}^{\infty} n^{-2} \sin ^{2}(n x) .
\end{gathered}
$$

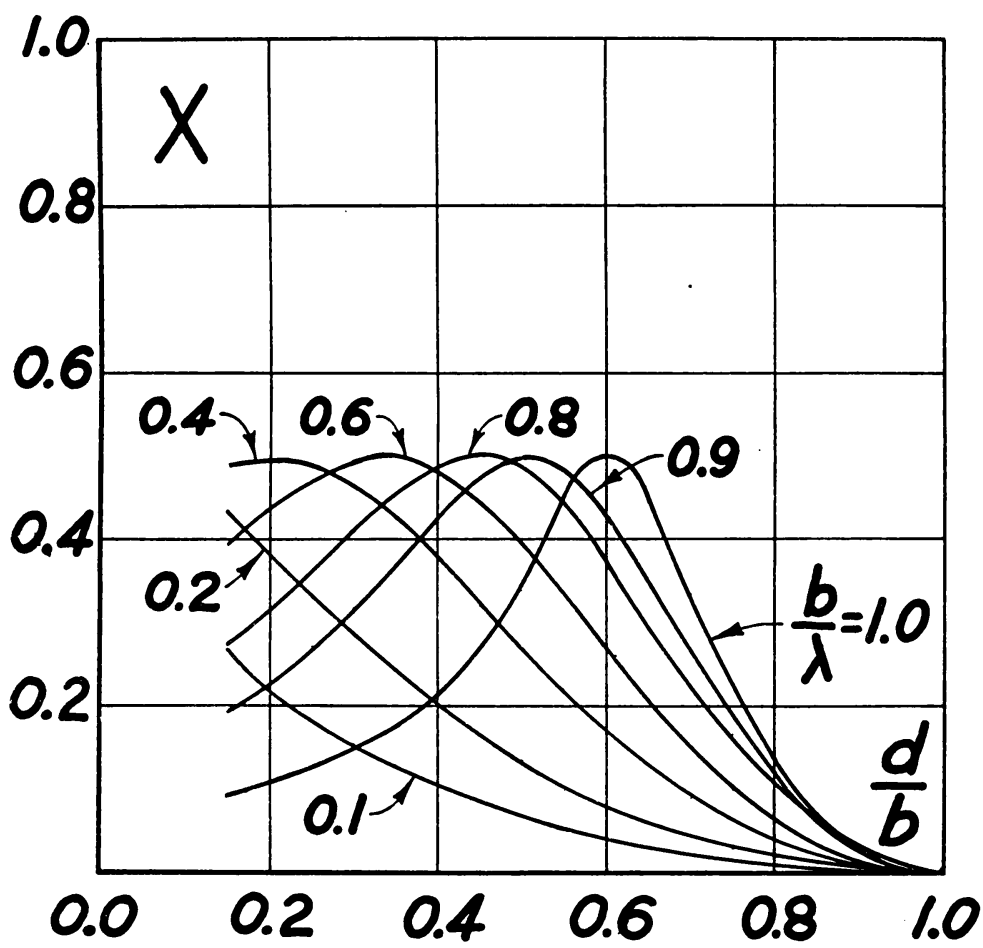

FIG. 4. Reactive component of grating impedance.

Differentiating Eq. (89) with respect to $x$ yields

$$
\begin{aligned}
& \frac{d S(x)}{d x}=\sum_{n=1}^{\infty} n^{-1} \sin (2 n x)=\operatorname{Im} \sum_{n=1}^{\infty} n^{-1} \exp (j 2 n x) \\
& =\operatorname{Im}\left[\ln \left(1-e^{i 2 x}\right)^{-1}\right]=\left(\frac{\pi}{2}-x\right) .
\end{aligned}
$$

Integrating Eq. (90) with respect to $x$, and requiring $S(0)=0$, yields

$$
S(x)=\frac{x}{2}(\pi-x) .
$$


Substituting Eq. (91) in Eq. (88) yields

$$
G=\frac{b}{d}
$$

Since $B$ is negligible

$$
T=\frac{1}{G}=\frac{d}{b} .
$$

in agreement with Kirchhoff's theory, so that Eq. (87) is certainly correct for sufficiently large values of $b / \lambda$. Eq. (93) is borne out by Fig. 5 .

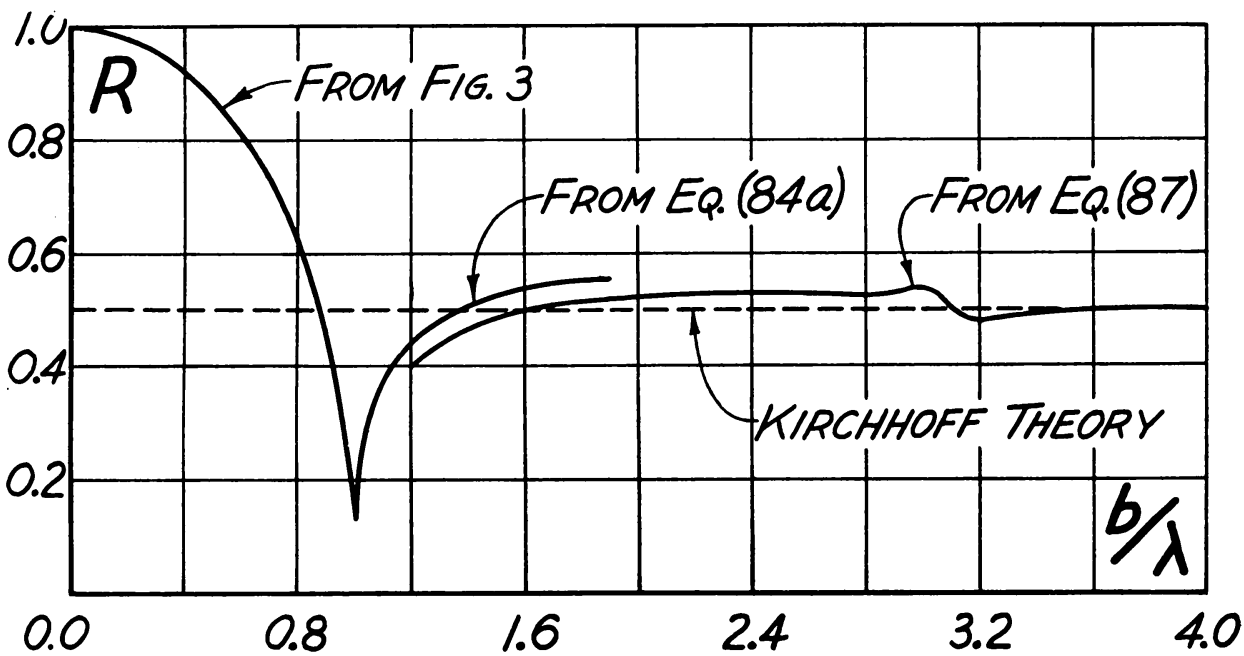

FIG. 5. Transmission coefficient for half open grating as computed from Eqs. (84a), (87), and Kirchhoff theory.

The value $T=d / b$ predicted by Kirchhoff's theory is plotted in Figs. 3 and 5 for comparison, and it is seen that the agreement is not good, even for values of $b / \lambda$ approaching 2 , but is evidently satisfactory for $b / \lambda>4$. Morse and Rubenstein, ${ }^{16}$ in calculating the exact results for the diffraction of a normally incident plane wave on a single slit, obtained agreement for values of $d / \lambda$ greater than about unity. On the other hand, Morse and Rubenstein's results show that it is necessary to go to quite large values of $b / \lambda$ in the case of oblique incidence before agreement with Kirchhoff's results is obtained. Unfortunately, the present method cannot be applied to the problem of oblique incidence for the grating, except perhaps in those special cases where the periodicity of the incident wave in a plane of constant $z$ is the same as the periodicity of the grating.

It is also of interest to note the sharp resonance at $b / \lambda=1$, in contrast to the slight $\operatorname{dip}$ at $b / \lambda=3$. There are no resonances at $b / \lambda=2$ or 4 due to the additional symmetry introduced at $d / b=1 / 2$.

${ }^{16} \mathrm{P}$. M. Morse and P. V. Rubenstein, The diffraction of waves by ribbons and slits, Phys. Rev. 54, 895-898 (1938). 